\title{
Do the Types of Seat Surface Influence the Pulmonary Functions during Prolonged Sitting?
}

\author{
Sung Min Son \\ Department of Physical Therapy, College of Health Science, Cheongju University, Cheongju, Korea
}

Purpose: The purpose of this study was to identify the effects of the types of seat surface (static or dynamic seat surface) on the pulmonary functions during prolonged sitting.

Methods: Thirty-four participants (20 males and 14 females) were recruited, and distributed randomly into dynamic prolonged sitting (DPS, $n=17$ ) and static prolonged sitting (SPS, $n=17)$ groups. The DPS group was seated on a chair with a dynamic air cushion, and the SPS group was seated on a chair without a dynamic air cushion. The pulmonary function was assessed before sitting, and after participants had been seated for one hour. The pulmonary function [forced vital capacity (FVC), forced expiratory volume in 1 second (FEV1), and Peak expiratory flow (PEF)] was measured using a spirometer.

Results: Statistical analyses revealed significant differences in the time $x$ group interactions of FVC, FEV1, PEF, and FEV1/FVC. The DPS group were significantly different in FVC, FEV1, PEF, and FEV1/FVC after prolonged sitting for one hour, compared to the SPS group $(\mathrm{p}<0.05)$.

Conclusion: These findings suggest that dynamic sitting can prevent a decrease in the physiological function, such as pulmonary functions, rather than static sitting during prolonged sitting.

Keywords: Prolonged sitting, Pulmonary function, Dynamic sitting

\section{서 론}

몸을 많이 움직이지 않는 행동(Sedentary behavior)은 거의 대부분 앉 은 자세 혹은 기대어 누운 자세(reclining posture)에서 이루어지며, 의 식이 깨어 있는 상태에서 낮은 에너지 소비가 이루어지는 행동학적 특성을 가지고 있다. 사무 작업, 자동차 운전, 텔레비전 시청과 같은 앉은 자세는 몸의 움직임이 줄어든 전형적인 자세로서 많은 사람들 이 앉은 자세를 유지하며 많은 시간을 보낸다. 하지만, 이와 같은 장 시간 앉은 자세(prolonged sitting)는 비만, 당뇨, 심혈관질환 등의 발생 을 증가시킬 수 있는 요인으로서 건강에 부정적으로 영향을 줄 수 있 다. ${ }^{3-5}$ 또한, 이전 연구들에서는 내과적인 부정적인 요인 이외에도 장 시간 앉은 자세를 유지할 경우 근골격계 질환의 발생 증가와 호흡 기 능에 악영향을 줄수 있다고 보고하였다. ${ }^{6.7}$

정상 성인의 호흡 기능에 영향을 줄 수 있는 요인은 다양하다. 허파 기능은 나이, 성별과 인종과 같은 요인뿐만 아니라 ${ }^{89}$ 다양한 신체의 자세에 따라차이가 날 수 있다. 동일한 앉은 자세일지라도 척추의 정
렬에 따라 호흡 능력의 변화가 나타날 수 있다.10,11 이전 선행 연구에서 는 슬럼프 앉기(slumped sitting), 척추 후만 앉은 자세(kyphotic sitting) 및 정상 앉기(normal sitting)와 같은 앉은 자세의 형태에 따라 허파 용 량(lung capacity)과 날숨 용적(expiratory volume)에서 차이가 있음을 확인하였다. ${ }^{2}$ 이와 같은 자세의 변화는 복부(abdomen) 내에 장기나 중력에 대한 가로막 움직임에 영향을 줄 수 있기 때문에 자세에 따라 호흡 능력이 변화 할 수 있다. 또한 Kang 등13의 연구에서는 앉은 자세 의 척추 정렬 상태만 아니라 장시간 앉은 자세를 유지할 경우에도 호 흡 기능의 감소가 나타남을 확인하였다.

장시간 앉은 자세를 유지할 경우 슬럼프 앉기나 척추 후만 앉기 자 세와 같이 척추 정렬이 부적절한 상태를 이루게 되어 체간의 움직임 이 부족해질 수 있으며, 이로 인해 체간 근육의 뺏뻣함(stiffness) 증가 와 관절움직임범위 감소와 같은 생체역학적인 변화가 발생 할 수 있 다.14,15 그래서, 오랜 시간 동안 앉은 자세를 유지할 경우 일반적인 앉 기보다는 자세를 다양하게 변화시킬 수 있는 앉기 방법이 필요하다. 동적 앉기(dynamic sitting; active sitting)는 똑바로 앉은 자세(upright
Received Dec 31, 2019 Revised Feb 13, 2020

Accepted Feb 13, 2020

Corresponding author Sung Min Son

E-mailssm0417@hanmail.net
Copylight (C2020 The Korean Society of Physical Therapy

This is an Open Access article distribute under the terms of the Creative Commons Attribution Non-commercial License (https:// creativecommons.org/license/by-nc/4.o.) which permits unrestricted non-commercial use, distribution, and reproduction in any medium, provided the original work is properly cited. 
sitting posture)를 유지하고 더 많은 체간의 움직임을 유도하기 위해 불안정한 지지면에서 앉는 것을 말한다. ${ }^{16}$ 이러한 동적 앉기는 짐볼 (gym ball)이나 의자의 지면 위에 에어쿠션(air cushion)과 같이 체중의 이동에 따라 앉은 지면이 순응하는 지면(compliant surface) 위에서 앉 은 자세를 유지할 때 나타난다. 선행 연구들에서 장시간 앉은 자세를 유지하는 동안 동적 앉기를 실시했을 때, 척추에 가해지는 압력 감소, 코어 근육(core muscle)의 수축 유도 및 신체 인식과 조절 증가와 같은 긍정적인 영향을 확인하였다.1718

이전 선행연구들에서는 단순히 장시간 앉은 자세 동안 호흡 기능과 같은 생리학적 변화나 균형 및 보행과 같은 기능적 능력의 변화를 확 인하였으며, 장시간 앉은 자세를 유지하는 동안 동적 앉기와 같은 앉은 지면의 형태에 따라 호흡 능력에 미치는 영향을 확인한 연구는 없었다. 그래서, 본 연구의 목적은 장시간 앉은 자세를 유지하는 동안 앉은 지 면의 형태에 따라 호흡 능력의 변화가 있는지 확인하는 것이다.

\section{연구 방법}

\section{1. 연구대상}

본 연구는 건강한 20 대 성인 34 명을 대상으로 하였다. 실험 전 설명을 듣고 자발적 참여를 동한 대상자들로만 실험을 진행하였다. 앉은 지 면의 형태에 따라 무작위 선정을 통해 동적 앉기 그룹( $\mathrm{n}=17)$ 과 정적 앉기 그룹( $\mathrm{n}=17)$ 으로 구분하였고, 각 그룹의 성별 비율은 각각 남자 10 명과 여자 7 명으로 구성하였다. 연구자는 실험 전 모든 대상자들에 게 본 연구에 대해 충분히 설명을 하였고, 모든 대상자들은 본 연구 의 설명을 듣고 목적을 이해하였다. 본 연구의 참여한 대상자의 선정 기준은 다음과 같다. 1) 양쪽 하지에 근골격계 혹은 신경학적 문제가 없는 자, 2) 심혈관계 질환 및 호흡기계에 문제가 없는 자, 3) 척추와 관 련한 질환이 없는 자로 하였다.

\section{2. 연구절차 및 측정도구}

1) 연구절차

일반적으로 장시간 앉기는 30 분 이상 다른 움직임이 최소화 된 상태 에서 앉기 자세를 유지하는 경우를 의미하며, ${ }^{19}$ 본 연구에서는 1 시간 동안 앉은 자세를 유지하는 동안 앉은 지면의 형태에 따른 호흡의 변 화를 측정하였다. 동적 앉기 그룹은 불안전한 지면을 유도하기 위해 의자 위에 다이나믹 에어큐션(Licensing Services International Inc., Philadelpia, PA, USA)을 의자 위에 놓고 진행하였으며, 정적 앉기 그룹 은 다이나믹 에어션 없이 의자에 앉은 상태로 실험을 진행하였다. 실 험에 사용된 의자는 등받이가 부착되지 않았으며, 의자 바닥 면이 미 끄러지지 않도록 미끄럼 방지 패드가 부착되었고, 대상자의 다리 길 이에 맞게 높이를 조절할 수 있는 의자로 하였다. 대상자들의 의자 높
이는 오금(popliteal) 높이로 설정하여 무릎 관절 각도가 $90^{\circ}$ 를 유지할 수 있도록 하였고, 두 발은 바닥에 붙이고 유지하도록 지시하였다. 추 가적으로 장시간 앉은 자세를 유지하는 동안 대상자들의 상지와 체 간은 편하게 위치하거나 편하게 자세를 취할 수 있도록 하였다. 장시 간 앉은 자세를 유지하는 동안 대상자의 눈높이에 맞춰 전방에 모니 터를 설치하여 동영상을 시청할 수 있도록 하였다.

\section{2) 자료수집}

중재 전-후 두 그룹의 호흡 기능을 평가하기 위해 폐 기능 측정 장비 (Micro Quark USB, Cosmed Ltd, Italy)를 사용하였다. 호흡 기능 측정 은 노력성 허파용량(forced vital capacity, FVC), 1초간 노력성 날숨용 적(forced expiratory volume in 1 second, FEV1)과 최대 날숨 유량(peak expiratory flow, $\mathrm{PEF}$ )를 측정하였다. 호흡기능을 측정하기 이전 대상 자들에게 검사 방법을 충분히 설명하였고, 검사자가 먼저 시범을 보 였으며 대상자들에게 2 번의 연습을 실시하였다. 호흡 기능 측정 자세 는 대상자를 오금 높이에 맞춘 의자에 앉아 시선은 정면을 보게 하였 고, 폐 기능 측정 장비의 마우스를 입으로 물고 검사자의 시작 신호에 맞추어 호흡을 하도록 하였다. $\mathrm{FVC}, \mathrm{FEV} 1$ 과 $\mathrm{FEF}$ 의 호흡 기능은 검사 자의 신호와 함께 평상시 호흡을 3-4회 정도 한 후 최대한 강하게 숨 을 들이미시고 최대한 빠르고 강하게 숨을 내쉬도록 하여 평가하였 다. 각 호흡 기능의 평가는 장시간 앉기 자세의 중재 전-후 평가하였 다. 중재 전-후 호흡 기능은 각각 3 번을 측정하여 평균값을 사용하였 고, 호흡 기능 평가 사이에 1 분간의 휴식을 주었다.

\section{3. 자료처리}

대상자들의 두 그룹의 일반적인 특성은 기술 통계와 독립 검정 t-test (independent t-test)를 통해 확인하였고, 정규성 검정은 KolmogorovSminov 검정을 실시하였다. 두 그룹의 장시간 앉은 자세 후 호흡 기능 의 변화를 평가하기 위해 이요인 반복측정 분산 분석(two-way repeated ANOVA)을 사용하였으며, 두 그룹 사이의 중재 효과를 비교하기 위해 독립 t-검정을 실시하였다. 수집 된 모든 측정값들의 자료 분석 은 SPSS 22.0 버전(IBM Co., Armonk, NY, USA)을 사용하였다. 통계학 적 유의 수준은 $\mathrm{p}<0.05$ 로 설정하였다.

\section{결 과}

본 연구에 참여한 대상자들은 정적 앉기 그룹과 동적 앉기 그룹 각 17 명씩 전체 34 명으로 구성되었으며, 두 그룹의 사이에 나이, 키와 몸 무게와 같은 일반적 특성에서 통계학적으로 유의한 차이는 없었다 ( $>$ >0.05) (Table 1). 장시간 앉기를 통한 두 그룹 사이의 중재 전-후의 호흡 기능 변화를 확인하기 위해 이요인 반복측정 분산 분석을 이용 
하였으며, $\mathrm{FVC}, \mathrm{FEV} 1, \mathrm{FEV1} / \mathrm{FEV}$ 과 $\mathrm{PEF}$ 에서 시간× 그룹에 대한 상호 작용이 모두 유의한 차이가 있었다. 시간에 대한 비교에서는 FVC 에 서만 유의한 차이가 있었으며, 다른 변수들에서는 유의한 차이가 없 었다. 두 그룹 사이에 대한 중재 효과 검정을 확인하기 위해 호흡 측 정 변수들의 전-후 차이값을 통해 비교하였고, 호흡 변수들 모두 (FVC, FEV1, FEV1/FEV, PEF에서)에서 유의한 차이가 있음을 확인하 였다(Table 2).

\section{고 찰}

많은 사람들은 다양한 일상생활활동과 업무를 위해 장시간 앉은 자 세를 유지하며, 이러한 장시간 앉은 자세는 호흡 기능에 저하를 가져 올 수 있다. 하지만, 동적 앉기 자세는 정적 앉기와 비교했을 때, 앉은 자세를 유지하는 동안 지속적으로 체간의 움직임을 유발할 수 있는 앉기 방식이다. 그래서, 우리의 연구는 앉은 지면의 형태가 장시간 앉 은 자세를 유지하는 동안 젊은 성인 대상자들의 호흡 능력에 차이가

Table 1. The general characteristics of subjects

\begin{tabular}{lccc}
\hline & $\begin{array}{c}\text { DPS group } \\
(\mathrm{n}=17)\end{array}$ & $\begin{array}{c}\text { SPS group } \\
(\mathrm{n}=17)\end{array}$ & $\mathrm{p}$ \\
\hline Male/Female & $10 / 7$ & $10 / 7$ & \\
Age (yr) & $20.59 \pm 1.28$ & $19.94 \pm 1.60$ & 0.202 \\
Height & $167.47 \pm 7.61$ & $169.00 \pm 9.58$ & 0.610 \\
Weight & $60.65 \pm 10.37$ & $65.06 \pm 13.16$ & 0.286 \\
\hline
\end{tabular}

Mean \pm SD.

DPS: dynamic prolonged sitting, SPS: static prolonged sitting.
있는지를 확인하기 위해 에어큐션을 사용한 동적 앉기 그룹과 일반 의자를 사용한 정적 앉기 그룹을 비교하였다. 본 연구의 결과에서 동 적 앉기 그룹은 중재 전-후에 호흡 능력의 차이는 거의 나타나지 않 았다. 하지만 정적 앉기 그룹에서는 중재-전후 호흡 능력의 감소가 뚜 렷하게 나타났으며, 두 그룹 간 비교에서도 정적 앉기 그룹에서 유의 한 호흡 능력의 감소의 감소가 나타났다. 따라서, 본 연구의 결과는 장 시간 앉은 자세를 유지할 경우 동적 앉기 자세가 호흡 기능과 같은 생 리적 요인에 긍정적으로 작용을 할 수 있는 앉기 방법임을 확인할 수 있는 연구였다.

우리 연구의 결과는 오랜 시간 정적 앉기를 유지할 경우 호흡 기능 에서 유의한 감소가 나타났으며, 동적 앉기에서는 중재 이전에 비해 호흡 기능에 거의 변화가 나타나지 않았다. Kang 등 ${ }^{13}$ 의 연구에서 장 시간 정적 앉기 자세를 취한 후 호흡 기능의 변화를 확인하였고, 이 연구의 결과에서 1 시간의 정적 앉기 후 전체적인 호흡 기능의 감소가 나타났다고 보고하였다. 이는 본 연구의 정적 앉기 그룹에서 나타난 결과와 동일하며, 본 연구의 결과를 뒷받침 한다고 할 수 있다. 장시간 앉은 자세는 여러 체간의 생체역학적인 요소들을 변화시킬 수 있으 며, 이러한 생체역학적인 변화는 호흡 기능에 부정적인 요인으로 작 용할 수 있다. 일반적으로 장시간 앉은 자세를 유지할 때 많은 사람들 은 무의식적으로 가장 편한 자세를 채택하게 되며, 체간이 슬럼프 된 자세를 취하게 된다. 이러한 슬럼프 된 자세로 오랜 시간을 유지할 경 우 척추 주위의 근육과 결합조직들에서도 시간에 따른 점탄성의 변 화로 주위 조직들의 뻣뻣함(stiffness)이 증가하거나 관절운동범위의 감소가 나타날 수 있다.,114,19,20 Beach 등 ${ }^{14}$ 의 연구에서도 장시간 동안 앉

Table 2. Comparison of pulmonary function in between the DPS and SPS group

\begin{tabular}{|c|c|c|c|c|c|}
\hline & Group & DPS $(n=17)$ & $\operatorname{SPS}(n=17)$ & Diff value & $p$ \\
\hline \multirow[t]{4}{*}{ FVC (L) } & Pre & $4.06 \pm 0.97$ & $4.10 \pm 0.80$ & $-0.025 \pm 0.11$ & \\
\hline & Post & $4.04 \pm 0.97$ & $3.98 \pm 0.83$ & $-0.124 \pm 0.14$ & $0.03^{*}$ \\
\hline & Time & & $0.02^{*}$ & & \\
\hline & Time $\times$ group & & $0.03^{*}$ & & \\
\hline \multirow[t]{4}{*}{ FEV1 (L) } & Pre & $3.29 \pm 0.77$ & $3.50 \pm 0.70$ & $0.057 \pm 0.12$ & \\
\hline & Post & $3.35 \pm 0.70$ & $3.35 \pm 0.77$ & $-0.148 \pm 0.21$ & $0.002^{*}$ \\
\hline & Time & & 0.135 & & \\
\hline & Time $\times$ group & & $0.002^{*}$ & & \\
\hline \multirow[t]{4}{*}{ FEV1/FVC (\%) } & Pre & $82.97 \pm 7.18$ & $85.29 \pm 4.81$ & $1.971 \pm 3.65$ & \\
\hline & Post & $84.94 \pm 7.34$ & $83.89 \pm 5.72$ & $-1.406 \pm 4.36$ & $0.02^{*}$ \\
\hline & Time & & 0.685 & & \\
\hline & Time $\times$ group & & $0.02^{*}$ & & \\
\hline \multirow[t]{4}{*}{$\mathrm{FEF}(\mathrm{L})$} & Pre & $6.80 \pm 1.96$ & $6.89 \pm 1.98$ & $0.062 \pm 0.37$ & \\
\hline & Post & $6.86 \pm 1.98$ & $6.69 \pm 2.11$ & $-0.201 \pm 0.36$ & $0.005^{*}$ \\
\hline & Time & & 0.281 & & \\
\hline & Time $\times$ group & & $0.046^{*}$ & & \\
\hline
\end{tabular}

Values are presented as mean \pm standard deviation. 
은 자세를 취한 후 허리뻐의 수동적인 움직임을 측정하였으며, 이 연 구의 결과에서 수동적인 굽힘 동작에서 움직임의 뺏뺏함이 증가하 였다 보고하였다. 우리의 연구 결과에서 나타난 장시간 정적 앉기 자 세에 따른 호흡 기능의 감소는 체간 주위의 근육과 주위결합조직들 의 변화로 인해 생체역학적인 움직임이 감소되어 나타난 결과라 생각 되어진다.

본 연구의 결과에서 정적 앉기 자세를 유지한 대상자와는 다르게 동적 앉기 자세를 실시한 대상자들에서는 장시간 앉은 자세를 유지 한 후에도 중재 전과 비교했을 때, 호흡 기능에 변화가 없거나 약간 향상된 수치를 보이고 있었다. 지금까지들의 선행 연구들에서 장시간 앉은 자세 혹은 장시간 앉은 자세를 유지하는 동안 지면의 형태에 따 른 호흡 기능의 영향에 대한 연구가 아직 많이 이루어지지 않아 어떤 기전에 의해 호흡 기능의 감소가 발생하는지 정확히 확인할 수는 없 다. 하지만, 동적 앉기가 정적 앉기에 비해 순응하는 지면의 형태가 체 중 중심을 이동시키면서 지속적으로 체간의 움직임을 유발시킬 수 있다는 것이다. 선행 연구들에서 동적 앉기 자세는 정적 앉기 자세에 비해 체중의 중심이 이동할 때 지면이 순응하는 불안정한 지지면에 서 장시간 앉은 자세를 취할 경우 지속적으로 척추의 움직임이 유발 할 수 있다고 하였다. ${ }^{21,22} \mathrm{Wang}$ 등의 연구에서도 장시간 동적 앉기는 체중 중심의 이동이 증가하였고, 지속적으로 척추 주위 근육내 기계 적 자극을 유발시킬 수 있다고 보고하였다. ${ }^{21}$ 이와 같은 지속적인 기 계적 자극의 입력은 신체 위치 인식과 자세 조절을 증가시킬 수 있다. Van Dieen 등 24 의 연구에서 표면 근전도 검사를 통해 동적 앉기 동안 체간의 지속적인 움직임을 유발할 수 있으며, 이는 Type I 근육의 이 완을 예방할 수 있다고 하였다. 또 다른 연구에서는 동적 앉기 자세는 코어근육[허리 다열근(lumbar multifidus), 배속빗근(internal oblique), 배바깥빗근(external oblique), 배곧은근(rectus abdomianl), 척추세움 근(erector spinae)]의 활성도를 높여 줄 수 있는 방법이라고 보고하였 다.1718 이러한 코어 근육은 호흡과 밀접과 관계가 있으며, 특히 배속빗 근과 배바깥빗근은 들숨과 날숨을 위해 중요한 역할을 하는 근육이 다. 본 연구의 결과에서 나타난 동적 앉기 그룹의 호흡 기능 유지는 불안정한 면에서 지속적인 체간의 움직임이 유발되어 주위 결합조직 들과 근육의 활성화가 유지된 결과라 사료된다.

우리의 연구 결과는 동적 앉기 자세는 지속적으로 척추 움직임을 유발시켜 체간 주위의 근육들의 수축을 유발시킬 수 있으며, 일상생 활활동에서 장시간 앉은 자세를 유지할 경우 정적 지면에서 앉은 자 세를 유지하는 것보다 동적 지면에서의 앉기를 하는 것이 호흡 기능 과 같은 생리학적 기능의 감소를 예방할 수 있음을 제시한다. 우리의 연구 결과를 토대로, 장시간 앉는 자세를 유지할 경우 일반적인 의자 에 앉는 것보다 에어큐션과 같은 체중의 이동에 따라 앉은 지면이 순 응하는 동적 앉기를 취하는 것이 호흡과 같은 생리학적 기능을 유지
하는데 더 효율적인 것이라 생각된다. 하지만, 우리의 연구에서 고려 해야 할 몇 가지 제한점을 가지고 있다. 첫째, 앉은 지면의 형태를 두 가지로만 비교하여 다양한 지면의 형태를 고려하지 못하였고, 1 시간 의 중재 후 즉각적인 효과만을 확인하여 시간의 흐름에 따른 변화를 확인하지 못하였다. 두번째, 실험에 참여한 대상자의 수가 적어 일반 화하기에는 어려움이 있다. 추가적으로 노인과 같은 호흡 기능의 변 화가 일상생활에 영향을 줄 수 있는 대상자로 연구가 이루어지지 않 았다는 것이다. 앞으로의 연구에서는 이러한 제한점을 보완한 추가 적인 연구가 계속적으로 이루어져야 할 것이다.

\section{REFERENCES}

1. Dunstan DW, Wiesner G, Eakin EG et al. Reducing office workers' sitting time: Rationale and study design for the stand up victoria cluster randomized trial. BMC Public Health. 2013;13:1057.

2. Milton K, Gale J, Stamatakis E et al. Trends in prolonged sitting time among european adults: 27 country analysis. Prev Med. 2015;77:11-6.

3. Chau JY, Grunseit AC, Chey T et al. Daily sitting time and all-cause mortality: a meta-analysis. PLoS One. 2013;8(11):e80000.

4. Lee IM, Shiroma EJ, Lobelo F et al. Effect of physical inactivity on major non-communicable diseases worldwide: An analysis of burden of disease and life expectancy. Lancet. 2012;380(9838):219-29.

5. Wilmot EG, Edwardson CL, Achana FA et al. Sedentary time in adults and the association with diabetes, cardiovascular disease and death: systematic review and meta-analysis. Diabetologia. 2012;55(11):2895-905.

6. Katz JN. Lumbar disc disorders and low-back pain: socioeconomic factors and consequences. J Bone Joint Surg Am. 2006;88 Suppl 2:21-4.

7. Waongenngarm P, Rajaratnam BS, Janwantanakul P. Perceived body discomfort and trunk muscle activity in three prolonged sitting postures. J Phys Ther Sci. 2015;27(7):2183-7.

8. Ostrowski S, Barud W. Factors influencing lung function: Are the predicted values for spirometry reliable enough? J Physiol Pharmacol. 2006; 57 Suppl 4:263-71.

9. Sebbane M, El Kamel M, Millot A et al. Effect of weight loss on postural changes in pulmonary function in obese subjects: a longitudinal study. Respir Care. 2015;60(7):992-9.

10. Albarrati A, Zafar H, Alghadir AH et al. Effect of upright and slouched sitting postures on the respiratory muscle strength in healthy young males. Biomed Res Int. 2018;2018:3058970.

11. Katz S, Arish N, Rokach A et al. The effect of body position on pulmonary function: a systematic review. BMC Pulm Med. 2018;18(1):159.

12. Lin F, Parthasarathy S, Taylor SJ et al. Effect of different sitting postures on lung capacity, expiratory flow, and lumbar lordosis. Arch Phys Med Rehabil. 2006;87(4):504-9.

13. Kang KW, Son SM, Ko YM. Time-varying changes in pulmonary function with exposure to prolonged sitting. Osong Public Health Res Perspect. 2016;7(6):382-4.

14. Beach TA, Parkinson RJ, Stothart JP et al. Effects of prolonged sitting on the passive flexion stiffness of the in vivo lumbar spine. Spine J. 2005; $5(2): 145-54$. 
15. Iatridis JC, MacLean JJ, Roughley PJ et al. Effects of mechanical loading on intervertebral disc metabolism in vivo. J Bone Joint Surg Am. 2006;88 Suppl 2:41-6.

16. Vera-Garcia FJ, Grenier SG, McGill SM. Abdominal muscle response during curl-ups on both stable and labile surfaces. Phys Ther. 2000; 80(6):564-9.

17. Dickin DC, Surowiec RK, Wang H. Energy expenditure and muscular activation patterns through active sitting on compliant surfaces. J Sport Health Sci. 2017;6(2):207-12.

18. O'Sullivan P, Dankaerts W, Burnett A et al. Lumbopelvic kinematics and trunk muscle activity during sitting on stable and unstable surfaces. J Orthop Sports Phys Ther. 2006;36(1):19-25.

19. Dunstan DW, Thorp AA, Healy GN. Prolonged sitting: is it a distinct coronary heart disease risk factor?. Curr Opin Cardiol. 2011 Sep;26(5): 412-9.
20. Dunk NM, Callaghan JP. Gender-based differences in postural responses to seated exposures. Clin Biomech (Bristol, Avon). 2005;20(10):110110.

21. Leivseth G, Drerup B. Spinal shrinkage during work in a sitting posture compared to work in a standing posture. Clin Biomech (Bristol, Avon). 1997;12(7-8):409-18.

22. Henry W, Kaitlyn JW, Mason C et al. The effect of active sitting on trunk motion. Journal of Sport and Health Science. 2014;3(4):333-7.

23. McGill SM, Kavcic NS, Harvey E. Sitting on a chair or an exercise ball: various perspectives to guide decision making. Clin Biomech (Bristol, Avon). 2006;21(4):353-60.

24. van Dieen JH, Oude Vrielink HH, Toussaint HM. An investigation into the relevance of the pattern of temporal activation with respect to erector spinae muscle endurance. Eur J Appl Physiol Occup Physiol. 1993;66(1): 70-5. 